\title{
Periaqueductal Gray Glutamatergic Transmission Governs Chronic Stress-Induced Depression
}

\author{
Yu-Cheng Ho', Tzer-Bin Lin², Ming-Chun Hsieh ${ }^{1,3}$, Cheng-Yuan Lai ${ }^{1,4}$, Dylan Chou' ${ }^{1,2}$, Yat-Pang Chau', \\ Gin-Den Chen ${ }^{5}$ and Hsien-Yu Peng,
}

'Department of Medicine, Mackay Medical College, New Taipei, Taiwan; ${ }^{2}$ Department of Physiology, School of Medicine, College of Medicine, Taipei Medical University, Taipei, Taiwan; ${ }^{3}$ Department of Physiology, College of Medicine, National Taiwan University, Taipei, Taiwan;

${ }^{4}$ Department of Veterinary Medicine, College of Veterinary Medicine, National Chung-Hsing University, Taichung, Taiwan; ${ }^{5}$ Department of

Obstetrics and Gynecology, Chung-Shan Medical University Hospital, Chung-Shan Medical University, Taichung, Taiwan

\begin{abstract}
The mechanisms underlying chronic stress-induced dysfunction of glutamatergic transmission that contribute to helplessness-associated depressive disorder are unknown. We investigated the relationship of $\alpha$-amino-3-hydroxy-5-methyl-4-isoxazolepropionic acid (AMPA) receptors and stress, and the neuroplastic changes of stress-induced depression-like behavior in the ventrolateral periaqueductal gray (vIPAG). We conducted whole-cell patch-clamp electrophysiological recordings in the VIPAG neurons. Depression-like behavior was assayed using tail suspension test and sucrose preference test. Surface and cytosolic glutamate receptor I (GluRI) AMPA receptor expression was analyzed using western blotting. Phosphorylated GluRI expression was quantified using western blotting and immunohistochemical analysis. Unpredictable inescapable foot shock stress caused reduction in glutamatergic transmission originating from both presynaptic and postsynaptic loci in the VIPAG that was associated with behavioral despair and anhedonia in chronic stress-induced depression. Pharmacological inhibition of GluRI function in the vIPAG caused depression-like behavior. Diminished glutamatergic transmission was due to reduced glutamate release presynaptically and enhanced GluR I-endocytosis from the cell surface postsynaptically. Chronic stress-induced neuroplastic changes and maladaptive behavior were reversed and mimicked by administration of glucocorticoid receptor (GR) antagonist and agonist, respectively. However, chronic stress did not affect $\gamma$-aminobutyric acid (GABA)-mediated inhibitory synaptic transmission in the VIPAG. These results demonstrate that depression-like behavior is associated with remarkable reduction in glutamatergic, but not GABAergic, transmission in the vIPAG. These neuroplastic changes and maladaptive behavior are attributed to GRdependent mechanisms. As reduced GluRI-associated responses in the VIPAG contribute to chronic stress-induced neuroplastic changes, this cellular mechanism may be a critical component in the pathogenesis of stress-associated neuropsychiatric disorders.

Neuropsychopharmacology (2018) 43, 302-312; doi:10.1038/npp.2017.199; published online II October 2017
\end{abstract}

\section{INTRODUCTION}

Major depressive disorder, affecting more than 120 million people worldwide every year, is a heterogeneous illness influenced by a variety of factors, including environmental stress-related factors (Duman et al, 2016). Subjects with repeated exposure to stressors have an elevated risk of depressive disorders (Wohleb et al, 2016). Although currently available antidepressant medications are the mainstay of treatment for patients with major depressive disorder, up to $60 \%$ of patients do not achieve adequate response following antidepressant therapy (Fava, 2003). This poor outcome is because of multiple subtypes and complex causes of major depressive disorders (Gerhard et al, 2016).

* Correspondence: Dr H-Y Peng, Department of Medicine, Mackay Medical College, No. 46, Section 3, Zhongzheng Road, Sanzhi District, New Taipei, 25245, Taiwan, Tel: +886 226360303 ext I239, Fax: +88622636 1295, E-mail: hsien.yu@gmail.com

Received 24 April 2017; revised 23 August 2017; accepted 25 August 2017; accepted article preview online 30 August 2017
Although neurochemical theory-based mechanisms have been intensively explored during the past several decades, neuroplasticity theory-oriented neurobiological mechanisms of depressive disorders remain elusive (Gerhard et al, 2016; Krystal et al, 2013).

The midbrain periaqueductal gray (PAG) is believed to be an essential part of the circuitry that participates in stressassociated behavior, such as defensive (Penzo et al, 2014; Tovote et al, 2016) and antinociceptive (Ho et al, 2013, 2015; Ho et al, 2011) behavior in response to threat. It integrates several inputs from various brain regions, such as the amygdala (Penzo et al, 2014), and sends excitatory outputs to the premotor targets in the medulla. The ventrolateral periaqueductal gray (vlPAG), a subdivision of the PAG, has been shown to be involved in not only defensive behavior such as freezing, flight, and analgesia (Ho et al, 2013, 2015; Ho et al, 2011; Tovote et al, 2016), but also stress-induced depression-like behavior (Johnson et al, 2016). A previous study demonstrated that activation of the vlPAG by electric stimulation initiates the expression of freezing behavior 
(Vianna et al, 2001). Recent reports indicated that the vlPAG receives direct anatomical and functional inputs originating from the central nucleus of the amygdala to control stressinduced maladaptive responses (Penzo et al, 2014). The vlPAG is, therefore, a critical core for stress-associated maladaptive behavior, including neuroplastic changes observed in chronic stress-induced psychiatric disorders, and for studying their underlying cellular mechanisms.

Evidence for the dysregulation of glutamatergic neurotransmission in patients with depression indicates that glutamate-mediated excitatory system is crucially involved in depressive disorders (Auer et al, 2000). It has been considered that dysfunction in the glutamatergic system in depression is related to the loss of postsynaptic-associated $\alpha$-amino-3-hydroxy-5-methyl-4-isoxazolepropionic acid (AMPA) receptors (Wei et al, 2016; Yuen et al, 2012) as well as reduction in presynaptic glutamate release in the prefrontal cortex (PFC) (Yuen et al, 2012). A previous study reported that repeated stress diminishes not only presynaptic glutamate release but also postsynaptic AMPA receptor expression in the PFC (Yuen et al, 2012), resulting in chronic stress triggered-cognitive impairment. Moreover, another recent study demonstrated that chronic stress specifically decreases AMPA receptor-mediated neuroplasticity in the hippocampus, thereby contributing to depression (Kallarackal et al, 2013). However, it is still largely unclear whether the vlPAG is involved in chronic stress-elicited depression-like helplessness responses. In the current study, we sought to explore the functional alterations and specific targets in the vlPAG and to reveal its potential role in chronic stress-induced depressive behavior.

\section{MATERIALS AND METHODS}

\section{Animal Preparations}

Adult male Sprague-Dawley rats weighing 200-250 g were used. They were housed at room temperature $\left(23 \pm 1^{\circ} \mathrm{C}\right)$ with a $12 \mathrm{~h}$ light/dark cycle (lights on from 0800 to $2000 \mathrm{~h}$ ) and were provided food and water ad libitum. All experimental procedures were performed in accordance with the policies and recommendations of the International Association for the Study of Pain and approved by the institutional animal care and use committee of Taipei Medical University, Taipei, Taiwan. All efforts were made to minimize the number of animals used.

\section{Establishment of the Learned Helplessness Model}

A well-established model of learned helplessness (LH) paradigm was used in the present study as described in previous studies (Hajszan et al, 2009) and are detailed in Supplementary Information.

\section{Sucrose Preference Test (SPT)}

Anhedonic behavior in rats was evaluated using the sucrose preference test and are detailed in Supplementary Information.
Tail Suspension Test (TST) and Forced Swim Test (FST)

Behavioral despair in rats was assayed using the tail suspension test and forced swim test and are detailed in Supplementary Information.

\section{Locomotor Activity and Nociceptive Behavioral Test}

Locomotor activity in rats was measured using the open field test. Nociceptive behavioral test in rats was assayed using the von Frey filament test and plantar test and are detailed in Supplementary Information.

\section{Whole-Cell Patch-Clamp Recordings}

Electrophysiological recordings were described as we published previously (Ho et al, 2011, 2013, 2015) and are detailed in Supplementary Information. All neurons were recorded from the ventrolateral subregion of PAG. Recordings were conducted blind to the rat groups.

\section{Western Blotting}

Western blot analyses of glutamate receptor 1 (GluR1) and phosphorylated GluR1 (pGluR1) were conducted on protein samples from the plasma membrane and subcellular fractions of rat vlPAG and are detailed in Supplementary Information.

\section{Immunofluorescence}

Immunofluorescence analyses of GluR1, pGluR1, and NeuN expression were performed on the vlPAG-containing slices and are detailed in Supplementary Information.

\section{Intra-vlPAG Microinjection}

Intra-vlPAG microinjection was performed as reported previously (Ho et al, 2015) and are detailed in Supplementary Information.

\section{Reagents and Vehicles}

See Supplementary Information.

\section{Statistical Analyses}

Data are expressed as mean \pm SEM. One-way analyses of variance (ANOVAs) were used to compare the results of electrophysiological recording data from three groups followed by post hoc Tukey's multiple comparison tests. A two-way ANOVA was used to analyze the current-voltage curve from different holding potential and firing rate, with the post hoc Bonferroni's multiple comparison test. Unpaired Student's $t$-tests were used for analyses between two groups. Differences were considered significant if $p<0.05$ was observed. 


\section{RESULTS}

\section{Chronic Stress Elicits LH Depression-Like Behavior}

To investigate the role of the vlPAG in mediating behavioral stress responses, we used rats that were subjected repeatedly to inescapable and unpredictable foot shock stress (Figures 1a and b) (Hajszan et al, 2009). After 6 days of the training procedure, rats exhibited high failure rate (Figure 1c; unpaired $t$-test, $p<0.01$ ) and prolonged escape latency (Figure 1d; unpaired $t$-test, $p<0.01$ ) from escapable foot shocks, a well-established depression-like phenotype.

a

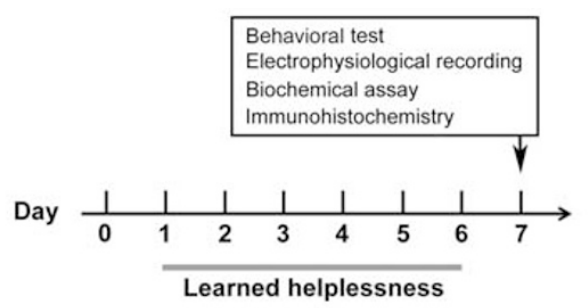

b

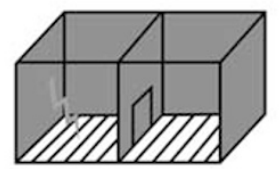

Training

60 shocks

$0.85 \mathrm{~mA}$

$15 \mathrm{sec}$ duration

$60 \mathrm{sec} \mid \mathrm{SI}$

C
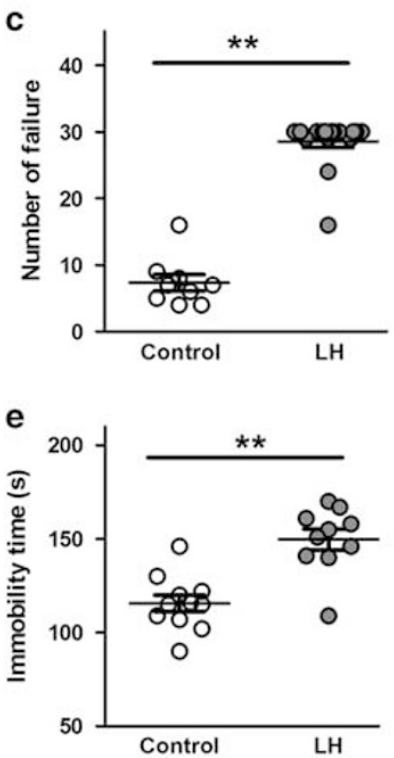

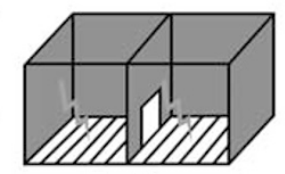

Testing

30 trials

$0.65 \mathrm{~mA}$

35 sec duration

$90 \mathrm{sec} \mid \mathrm{SI}$
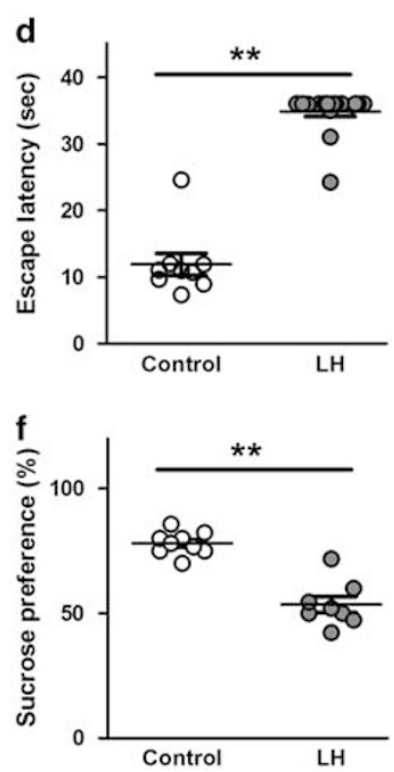

Figure I Inescapable unpredictable foot shock stress elicits helplessness behavior and depression-like behavior. (a) Schematic experimental procedure of learned helplessness $(\mathrm{LH})$ model. (b) Training and testing protocol of LH model. (c, d) Vertical scatterplots depicting the distribution of helpless behaviors, number of failure (c) and escape latency (d), from control $(n=9)$ and LH groups $(n=16)$. (e) Vertical scatterplot depicting the distribution of dispirited behavior, immobility time of tail suspension test, from control $(n=\mid \mathrm{I})$ and $\mathrm{LH}(n=\mid 0)$ groups. ( $f$ Vertical scatterplot depicting the distribution of anhedonic behavior, sucrose consumption of sucrose preference test, from control $(n=9)$ and $\mathrm{LH}(n=8)$ groups. Data are expressed as mean $\pm \mathrm{SEM}$; $* * * 0.01$ vs Control.

This suggests LH was successfully established by chronic inescapable and unpredictable stress (Li et al, 2011; Maier, 1984). In addition to the reduction in escape responses, $\mathrm{LH}$ rats also displayed an increase in immobile time during the TST (Figure 1e; unpaired $t$-test, $p<0.01$ ) and a reduction in sucrose consumption (Figure 1f; unpaired $t$-test, $p<0.01$ ), an indication of dispirited behavior and anhedonia behavior, respectively. Furthermore, $\mathrm{LH}$ rats exhibited reductions in paw withdrawal threshold during the von Frey test (Supplementary Figure S1a; unpaired t-test, $p<0.01$ ) and in paw withdrawal latency during plantar test (Supplementary Figure S1b; unpaired $t$-test, $p<0.01$ ). The above-mentioned results show that rats that received $\mathrm{LH}$ training successfully exhibit depression-like behavior and behavioral hypersensitivity.

\section{Chronic Stress Leads to Hypofunction of Glutamatergic Transmission onto PAG Neurons through Pre- and Postsynaptic Mechanisms}

The vlPAG has been reported as a crucial midbrain nucleus controlling various forms of stress-related behavior including freezing (Penzo et al, 2014; Tovote et al, 2016) and antinociceptive (Ho et al, 2011, 2013, 2015) responses. Activation of glutamatergic transmission in the vlPAG leads to stress-related antinociception by sending inhibitory outputs to the spinal dorsal horn (Heinricher et al, 2009; Lee et al, 2016; Ossipov et al, 2010). Therefore, we first examined whether inescapable foot shock stress alters glutamatergic transmission onto vlPAG neurons, resulting in depressionlike behavior, by conducting acute brain slices electrophysiological recordings. We assessed the current-voltage relationship of excitatory postsynaptic AMPA currents $\left(\right.$ EPSC $\left._{\mathrm{AMPA}} \mathrm{s}\right)$ in the vlPAG neurons evoked by electrical stimulation at various holding potentials. The amplitudes of EPSC $_{\mathrm{AMPA}} \mathrm{s}$ of vlPAG neurons in control rats were reduced at positive membrane potentials. In contrast, the EPSC $_{\mathrm{AMPA}} \mathrm{S}$ recorded from LH rats displayed a near-linear currentvoltage relationship (Figure 2a; two-way ANOVA, $\left.\mathrm{F}_{7,63}=2.632, \quad p<0.05\right)$. This suggests that calciumpermeable AMPA receptor-mediated responses in the vlPAG neurons might participate in chronic stress-induced depression-like behavior. We also found that synaptic strengths in the $\mathrm{LH}$ rats were drastically reduced compared with the control rats, revealed by reduction in the $\mathrm{EPSC}_{\mathrm{AMPA} / \mathrm{NMDA}}$ ratio (NMDA: $N$-methyl-D-aspartate; Supplementary Figure S2; unpaired $t$-test, $p<0.01)$. Next, to determine whether the decreased AMPA receptor-mediated responses originate from presynaptic, postsynaptic, or both loci, we conducted the paired-pulse ratio (PPR) experiments in both groups. Interestingly, we found that vlPAG neurons from $\mathrm{LH}$ rats exhibited higher PPR than did vIPAG neurons from the control group (Figure $2 \mathrm{~b}$; unpaired $t$-test, $p<0.01$ ), suggesting that a presynaptic change might modulate glutamatergic transmission induced by LH procedure. Moreover, we further investigated whether postsynaptic AMPA receptors were altered after the LH paradigm. Bath application of AMPA, an agonist of AMPA receptor, produced significantly smaller inward currents in the LH group compared with the control group (Figure 2c; unpaired $t$-test, $p<0.01$ ), implying that downregulation of postsynaptic AMPA receptors might occur in chronic stress-induced depression-like behavior. 
a

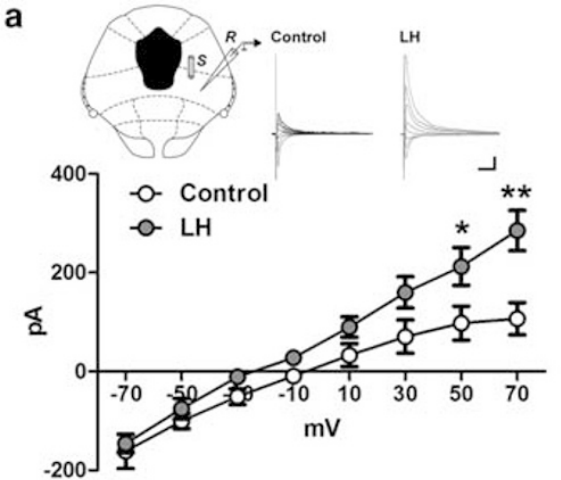

b

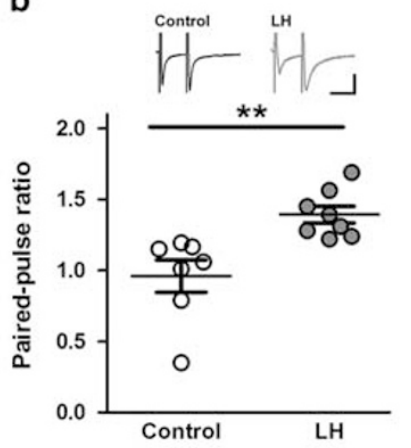

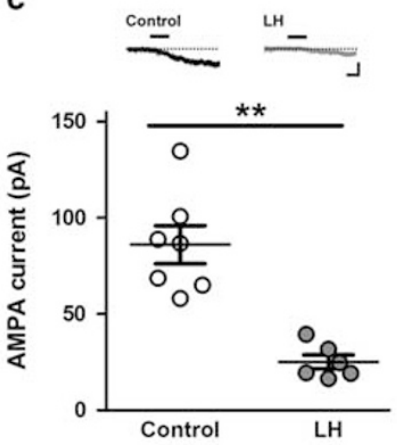

d

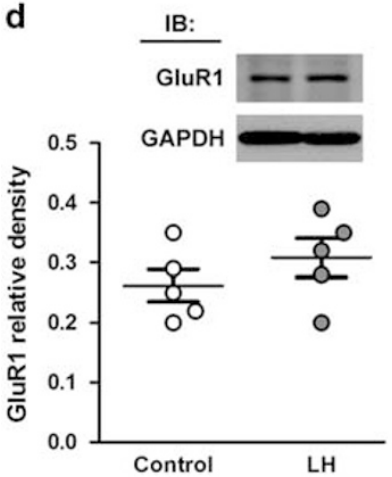

e

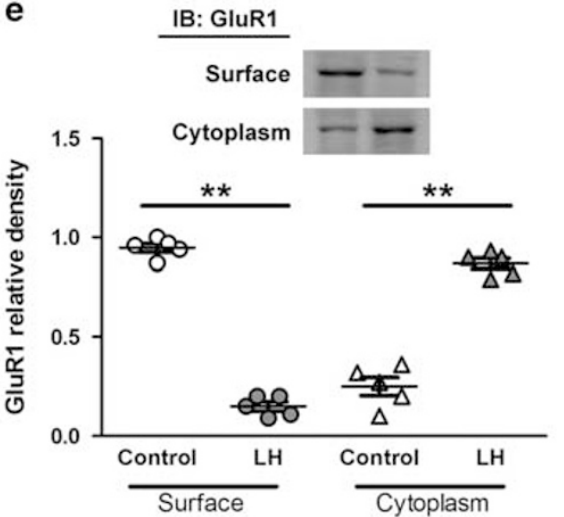

f

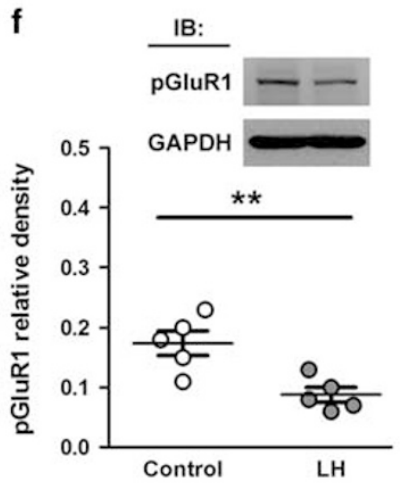

g

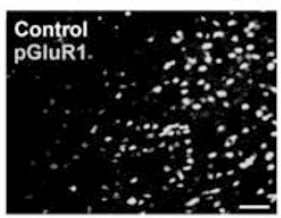

LH

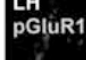

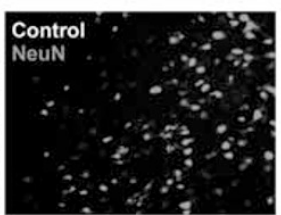

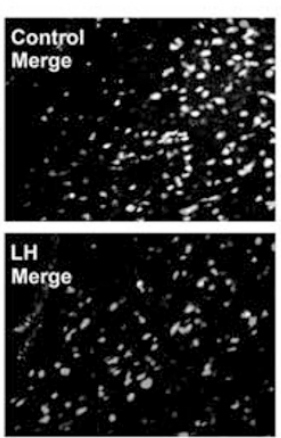

h

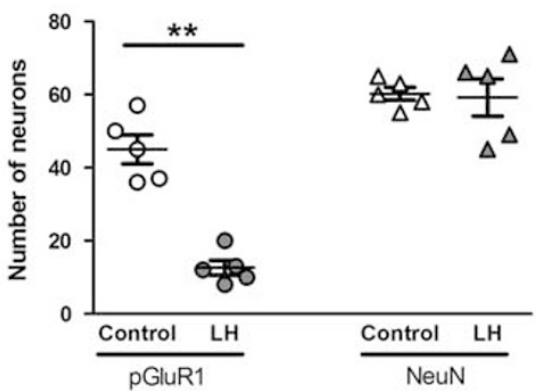

Figure 2 Chronic stress leads to depression of excitatory synaptic transmission and pGluRI protein expression in the ventrolateral periaqueductal gray (vIPAG). (a) The current-voltage (I-V) curves of EPSC AMPAS of VIPAG neurons recorded at holding potentials ranging from -70 to $+70 \mathrm{mV}$ in control and LH groups. Left inset: a diagram illustrating the positions of the stimulating $(S)$ and recording $(R)$ electrodes, respectively, in the vIPAG slice. Right inset:

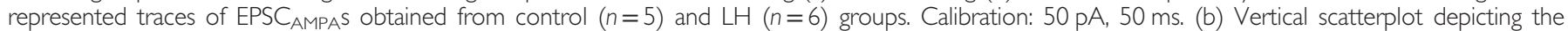
distribution of paired-pulse ratio (PPR) evoked by two identical electric stimuli with 50 ms interpulse interval. Inset: represented traces of PPR obtained from control $(n=7)$ and LH $(n=8)$ groups. Calibration: 50 pA, 50 ms. (c) Vertical scatterplot depicting the distribution of AMPA (I HM)-induced inward currents in control $(n=7)$ and LH $(n=6)$ groups. Horizontal bars denote the periods that AMPA were applied. The dashed line indicates the baseline. Calibration: 50 pA, I min. ( $d-f$ ) Vertical scatterplots depicting the distribution of total GluRI (d), surface and cytoplasm (e), and pGluRI (f) expressions in the vIPAG. Inset: represented western blot of protein expression from control $(n=5)$ and $\mathrm{LH}(n=5)$ groups. $(\mathrm{g}, \mathrm{h})$ Immunohistochemistry images (g) and quantitative analysis (h) of pGluRI and NeuN in the vIPAG slices dissected from control $(n=5)$ and LH $(n=5)$ groups. Calibration: $50 \mu m$. Data are expressed as mean \pm SEM; $* p<0.0$ I, *** $<0.01$ vs Control.

Calcium-permeable AMPA receptors are a determinant factor in the generation of some forms of neuroplastic changes in the central nervous system (Park et al, 2016). Dysfunctions of postsynaptic calcium-permeable AMPA receptors, such as GluR1 AMPA receptor, have been evidenced as involved in stress-induced neuropsychiatric disorders (Wei et al, 2016; Yuen et al, 2012). Hence, here we estimated the protein expression of GluR1, the main subunit of calcium-permeable AMPA receptors for excitatory transmission, in the brain. Unexpectedly, we found no significant difference in GluR1 protein expression level (Figure 2d; $p=0.306$ ) and number of neurons expressing GluR1 (Supplementary Figure S3; unpaired $t$-test, $p=0.78$ ) in the vlPAG after the LH paradigm. Based on a previous study, this may result from AMPA receptor mobilization between the cell surface and cytoplasm (Shi et al, 1999). Therefore, we isolated the membrane and cytosolic fractions to further investigate the distribution of GluR1 protein expression. We 

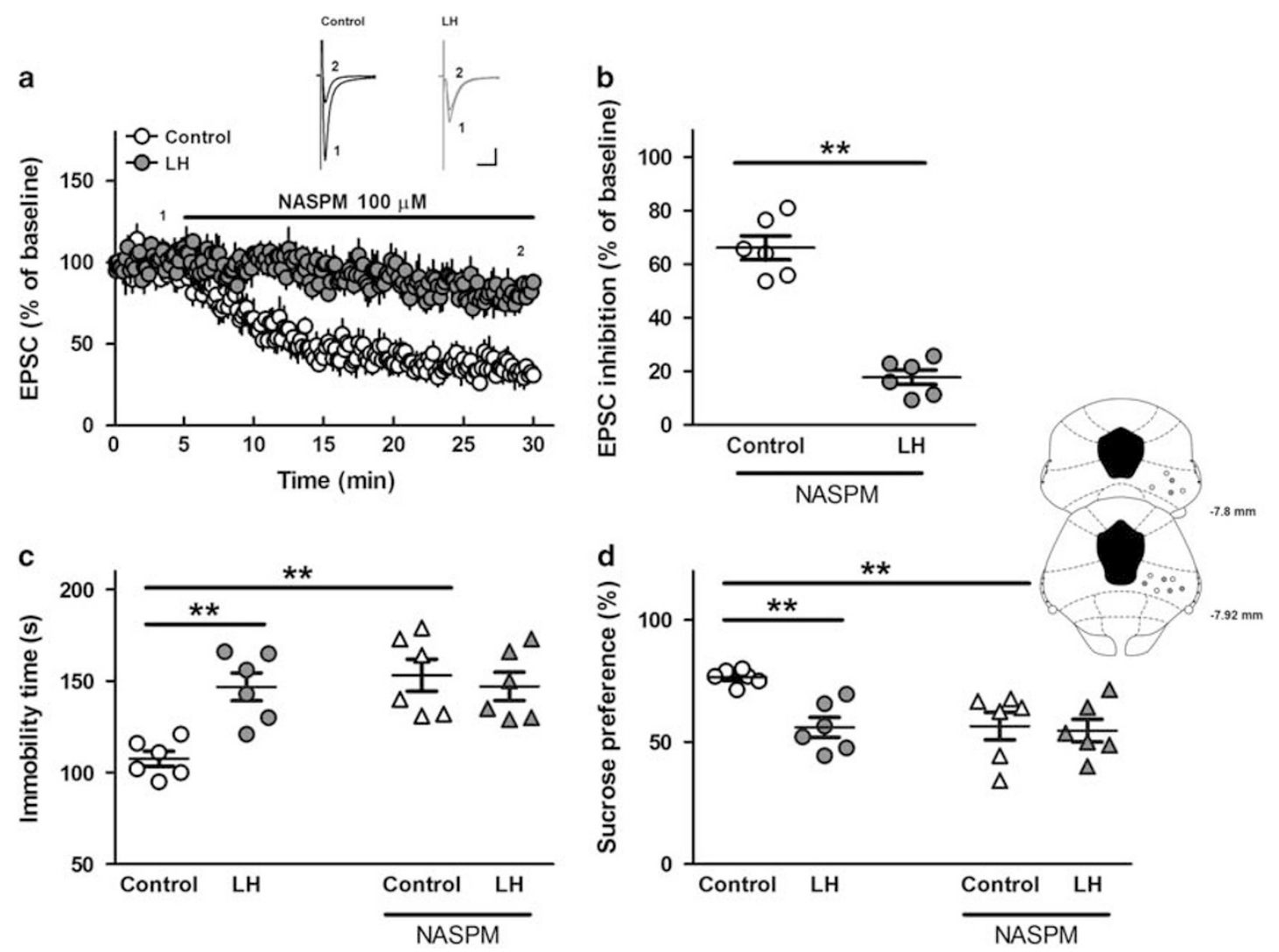

Figure 3 Inhibition of calcium-permeable GluRI AMPA receptor in the vIPAG causes depression-like behavior. (a) Effect of bath application of NASPM $($ I $00 \mathrm{MM})$ on the amplitude of EPSCs in VIPAG neurons dissected from control and LH groups. Horizontal bar denotes the periods that NASPM was applied. Inset: representative traces recorded at the time points denoted by the numbers on the time-course graph. Calibration: 50 pA, 20 ms. (b) Vertical scatterplot depicting the distribution of NASPM-induced EPSC inhibition recorded from vIPAG neurons obtained from control $(n=6)$ and LH $(n=6)$ groups. (c) Vertical scatterplot depicting the distribution of immobility time of tail suspension test, from control $(n=6)$ and $\mathrm{LH}(n=6)$ groups before (circle) and after (triangle) intra-vIPAG microinjection of NASPM. (d) Vertical scatterplot depicting the distribution of sucrose consumption of sucrose preference test, from control $(n=6)$ and $\mathrm{LH}(n=6)$ groups before (circle) and after (triangle) intra-vIPAG microinjection of NASPM. Inset: cannula tip placements for rats infused with NASPM in control (open circle) and LH (filled circle) groups. Data are expressed as mean \pm SEM; ** $p<0.0$ I vs Control.

found that LH caused decreased GluR1 expression on the cell surface and increased GluR1 expression in the cytoplasm as compared with the controls (Figure 2e; unpaired $t$-test, $p<0.01)$. This suggests that redistribution, but not overall increase in the quantity, of GluR1 expression may be caused by LH. A previous report indicated that phosphorylation of GluR1 on the serine 845 site is essential for AMPA receptor mobilization (Lee et al, 2003). Given that, we further identified whether LH reduced surface GluR1 expression by quantification of pGluR1 level. Anticipatedly, reduced pGluR1 protein expression was found in the vlPAG after the LH paradigm (Figure 2f; unpaired $t$-test, $p<0.01$ ). Furthermore, neurons expressing pGluR1 in the vlPAG were observed using immunohistochemical analyses in both groups (Figure 2g). However, reduced pGluR1 expression (Figure $2 \mathrm{~h}$; unpaired $t$-test, $p<0.01$ ), but not neuronal numbers (Figure $2 \mathrm{~h}$; unpaired $t$-test, $p=0.858$ ), was observed in the LH group, demonstrating that chronic stress may reduce phosphorylation-dependent expression of surface GluR1 in the vlPAG.

Moreover, we studied the passive membrane properties of vlPAG neurons in both groups. Neither membrane capacitance nor resistance was significantly different in the control and LH groups (Supplementary Figure S4). Furthermore, there were no significant differences in the resting membrane potentials in both the control and LH groups (Supplementary Figure S4). These results suggested that LH does not alter the passive membrane properties of vlPAG neurons. In addition, we also examined the neuronal excitability of vlPAG and dlPAG neurons by current injection experiment. We found that vlPAG neurons exhibited fewer spike numbers in the LH group compared with the control group (Supplementary Figures S5a and c; two-way ANOVA, $\mathrm{F}_{10,230}=4.353, p<0.01$ ), suggesting that chronic stress reduced vlPAG neuronal excitability. In contrast to vlPAG neurons, dlPAG neurons displayed higher firing frequency in the LH group compared with the control group (Supplementary Figures S5b and d; two-way ANOVA, $\left.\mathrm{F}_{10,160}=6.51, p<0.01\right)$, demonstrating that chronic stress enhanced dIPAG neuronal excitability. These results demonstrated that chronic stress reduced neuronal excitability in the vlPAG but not dlPAG neurons.

\section{Inhibition of Calcium-Permeable GluR1 AMPA Receptor in the vlPAG Contributes to Depression-Like Behavior}

Our results showed that neurons from the control group showed inward rectification, implying that calcium- 
permeable AMPA receptors, GluR1, might be involved in synaptic EPSC $_{A M P A} S$ (Figure 2a). In contrast, EPSC AMPA $_{A}$ recorded from the LH rats have a characteristic near-linear current-voltage relationship. This suggested that synaptic EPSC $_{\mathrm{AMPA}} \mathrm{s}$ might be mediated predominantly by GluR1 AMPA receptors. Therefore, to determine whether reduced GluR1 AMPA receptor function in the vlPAG contributes to chronic stress-induced diminished glutamatergic transmission, we used NASPM (Martinez-Rivera et al, 2017; Nishitani et al, 2014), a selective antagonist of calcium-permeable AMPA receptors. Inhibition of glutamatergic transmission by NASPM was higher in neurons from the control group than in neurons from the LH group (Figures $3 a$ and $b$; unpaired $t$-test, $p<0.01)$. This suggested that chronic stress decreased the prevalence of GluR1 calcium-permeable AMPA receptors in the vlPAG. Moreover, intra-vlPAG microinjection of NASPM increased TST and decreased SPT in control group (Figure 3c; one-way ANOVA, $\mathrm{F}_{3,20}=8.318$, $p<0.01$ and Figure 3d; one-way ANOVA, $\mathrm{F}_{3,20}=6.065$, $p<0.01)$. This suggested that inhibition of GluR1 calciumpermeable AMPA receptors in the vlPAG contributed to depression-like behavior. a
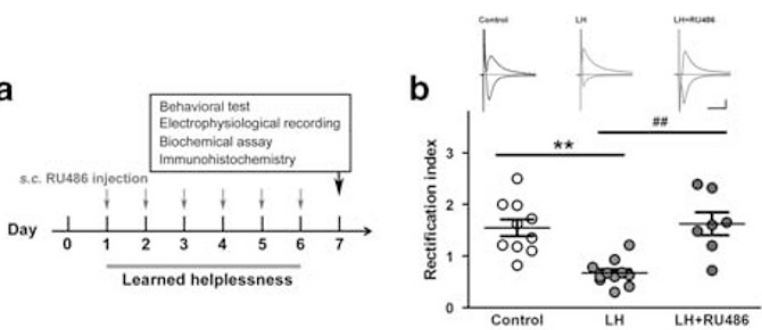

$\mathbf{C}_{\text {control }}$

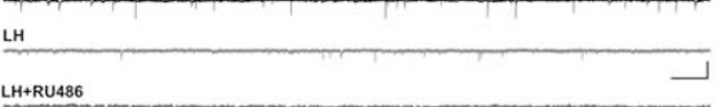

d
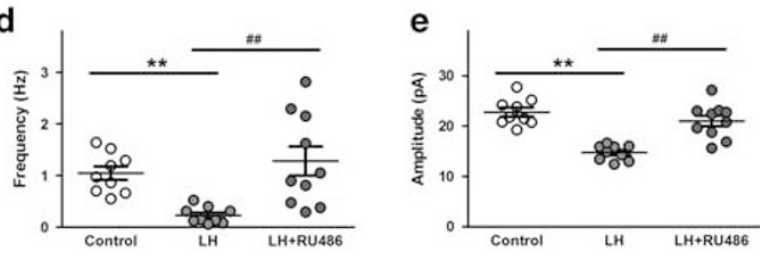

f

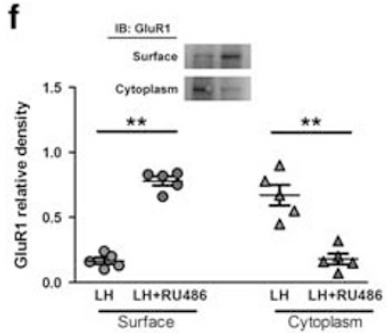

g

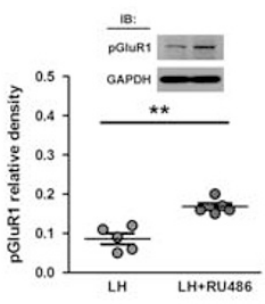

h
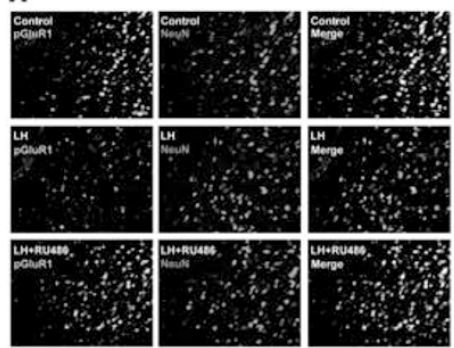

i

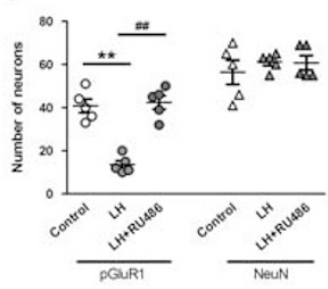

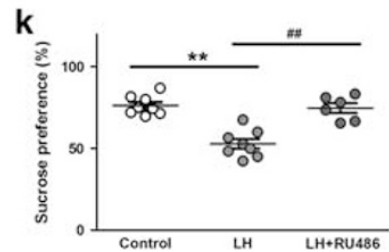

\section{Chronic Stress Causes Hypofunction of GluR1 in the vlPAG via Glucocorticoid Receptor-Dependent Mechanism}

To determine how chronic stress diminishes the functions of GluR1 in repeatedly stressed animals, we examined the involvement of the glucocorticoid receptor (GR) as GR activation initiates the reduction of AMPA receptor function contributing to cognitive impairment (Wei et al, 2016). To block the effects of GR, rats received daily subcutaneous injection of RU486 (10 mg/kg), a GR antagonist, $30 \mathrm{~min}$ before the LH paradigm (Figure 4a). Daily GR antagonist injection prevented chronic stress-induced decreased rectification index (Figure 4b; one-way ANOVA, $\mathrm{F}_{2,25}=13.78$, $p<0.01$ ), frequency (Figure $4 \mathrm{~d}$; one-way ANOVA, $\mathrm{F}_{2,25}=8.22, p<0.01$ ), and amplitude (Figure 4e; one-way ANOVA, $\left.\quad F_{2,25}=22.89, p<0.01\right)$ of miniature EPSCs (mEPSCs) in vlPAG neurons. Compared with control group (variance $\left(\sigma^{2}\right)=0.15$ ), LH+RU486 (variance $\left.\left(\sigma^{2}\right)=0.79\right)$ accompanies with much greater variance. Moreover, increased burst-like events were found in the vlPAG neurons after daily GR antagonist injection (baseline frequency: $1.28 \pm 0.28 \mathrm{~Hz}$ vs burst-like event frequency: $47.24 \pm 4.87 \mathrm{~Hz}$, unpaired $t$-test, $p<0.01$; Figure $4 \mathrm{c}$, lower panel). In addition, not only surface GluR1 but also pGluR1 expression was enhanced by daily subcutaneous RU486

Figure 4 Chronic stress diminishes excitatory synaptic transmission through both pre- and postsynaptic mechanisms in a glucocorticoid receptor (GR)-dependent manner. (a) Schematic experimental procedure of the effect of glucocorticoid receptor antagonist, RU486, in LH. (b) Rectification index (EPSC $-70 \mathrm{mv} / \mathrm{EPSC}_{+70 \mathrm{mV}}$ ) of AMPA receptor obtained from control $(n=10), \mathrm{LH}(n=1 \mathrm{I})$, and LH+RU486 $(n=7)$ groups. Inset: represented traces of rectification index obtained from control and $\mathrm{LH}$ groups. Calibration: $50 \mathrm{pA}, 20 \mathrm{~ms}$. (c) Representative traces of mEPSCs recorded from vIPAG neurons dissected from control, $\mathrm{LH}$, and $\mathrm{LH}+\mathrm{RU} 486$ groups. Calibration: 25 pA, 600 ms. (d, e) Vertical scatterplots depicting the distribution of frequency (d) and (e) amplitude of mEPSCs recorded from vIPAG neurons obtained from control $(n=9), \mathrm{LH}(n=9)$, and $\mathrm{LH}+\mathrm{RU} 486$ $(n=10)$ groups. ( $f, g)$ Vertical scatterplot depicting the distribution of surface and cytoplasm ( $\mathrm{f}$ ) and pGluRI (g) expressions in the vIPAG. Inset: represented western blot of protein expression from $\mathrm{LH}(n=5)$ and $\mathrm{LH}+$ RU486 ( $n=5)$ groups. ( $h, i)$ Immunohistochemistry images (h) and quantitative analysis (i) of pGluRI and NeuN in the VIPAG slices dissected from control $(n=5), \mathrm{LH}(n=5)$, and LH+RU486 $(n=5)$ groups. Calibration: $50 \mu \mathrm{m}$. (j, k) Vertical scatterplot depicting the distribution of immobility time of tail suspension test (j) and sucrose consumption of sucrose preference test $(k)$ from control $(n=8), \mathrm{LH}(n=8)$, and $\mathrm{LH}+\mathrm{RU} 486(n=6)$ groups. Data are expressed as mean \pm SEM; ${ }^{*} * x<0.01$ vs Control, ${ }^{\# \#} p<0.01$ vs $\mathrm{LH}$. 
injection (Figures 4f and g; unpaired $t$-test, $p<0.01$ ). The reversal effects of RU486 were also revealed by immunohistochemical results in vitro (Figures $4 \mathrm{~h}$ and i; one-way ANOVA, $F_{2,12}=34.08, p<0.01$ ) and behavioral results in vivo including TST, SPT, and FST (Figure 4j; one-way ANOVA, $\mathrm{F}_{2,19}=9.906, p<0.01,4 \mathrm{~K}$; one-way ANOVA, $\mathrm{F}_{2,19}=25.20$, $p<0.01$, and Supplementary Figure S6; one-way ANOVA, $\mathrm{F}_{2,15}=18.64, p<0.01$ ), respectively. These results demonstrated that chronic stress increased endocytosis of postsynaptic GluR1 through GR-dependent mechanism.

To clarify the role of GR in the chronic stress-induced hypofunction of glutamatergic transmission and depressionlike behavior, we generated naive rats that received subcutaneous injection of dexamethasone (DEX) $(2 \mathrm{mg} / \mathrm{kg})$ daily (Figure 5a), a selective glucocorticoid receptor agonist, to activate GR exogenously and mimic chronic stressinduced depression-like behavior. Rats that received subcutaneous injection of DEX had reduced body weight gain per se (Supplementary Figure S7a; unpaired $t$-test, $p<0.01$ ). As expected, DEX-treated rats exhibited decreased rectification index (Figure 5b; unpaired $t$-test, $p<0.01$ ), frequency (Figure 5d; unpaired $t$-test, $p<0.01$ ), and amplitude (Figure 5e; unpaired $t$-test, $p<0.01$ ) of mEPSCs in the vlPAG neurons compared with the control group. Moreover, DEX also diminished both surface GluR1 and pGluR1 protein expression in the vlPAG (Figures $5 f$ and g; unpaired $t$-test, $p<0.01)$. Immunohistochemical evidence also demonstrated that pGluR1, but not NeuN, expression was reduced by daily DEX injection (Figures $5 \mathrm{~h}$ and i; unpaired $t$-test, $p<0.01)$. Moreover, rats that received daily subcutaneous injection of DEX had increased TST and decreased SPT (Figures $5 \mathrm{j}$ and $\mathrm{k}$; unpaired $t$-test, $p<0.01$ ). In addition, DEX also suppressed locomotor activity (Supplementary Figures S7b and c; unpaired $t$-test, $p<0.01$ ) and time in central zone (Supplementary Figures S7b and d; unpaired $t$-test, $p<0.01$ ) of open field test. The above-mentioned results confirmed that GR activation is required for both the downregulation of GluR1 on the cell surface of vlPAG neurons and depressionlike behavior after chronic stress.

\section{Chronic Stress Does Not Affect GABAergic Transmission in the vlPAG}

Alteration in GABAergic transmission leads to the pathogenesis of major depressive disorder (Luscher et al, 2011). In addition to glutamatergic excitatory transmission in the vlPAG, GABAergic-mediated inhibitory transmission also plays a critical role in controlling the excitability of vlPAG neurons (Aubrey et al, 2017; Lau and Vaughan, 2014). Hence, we investigated whether chronic stress altered GABAergic transmission in the vlPAG causing depressionlike behavior. We evaluated the PPR of GABAergic transmission and miniature IPSC (mIPSC) recordings to identify whether chronic stress alters GABAergic-mediated transmission in the vlPAG. We observed no significant differences in the PPR between the control and LH groups (Supplementary Figure S8b; unpaired t-test, $p=0.742$ ). Moreover, we found that neither the frequency (Supplementary Figures S8c and d; unpaired $t$-test, $p=0.517$ ) nor the amplitude (Supplementary Figures S8c and e; unpaired $t$-test, $p=0.092$ ) of mIPSCs recorded in vlPAG slices from the LH group were significantly different
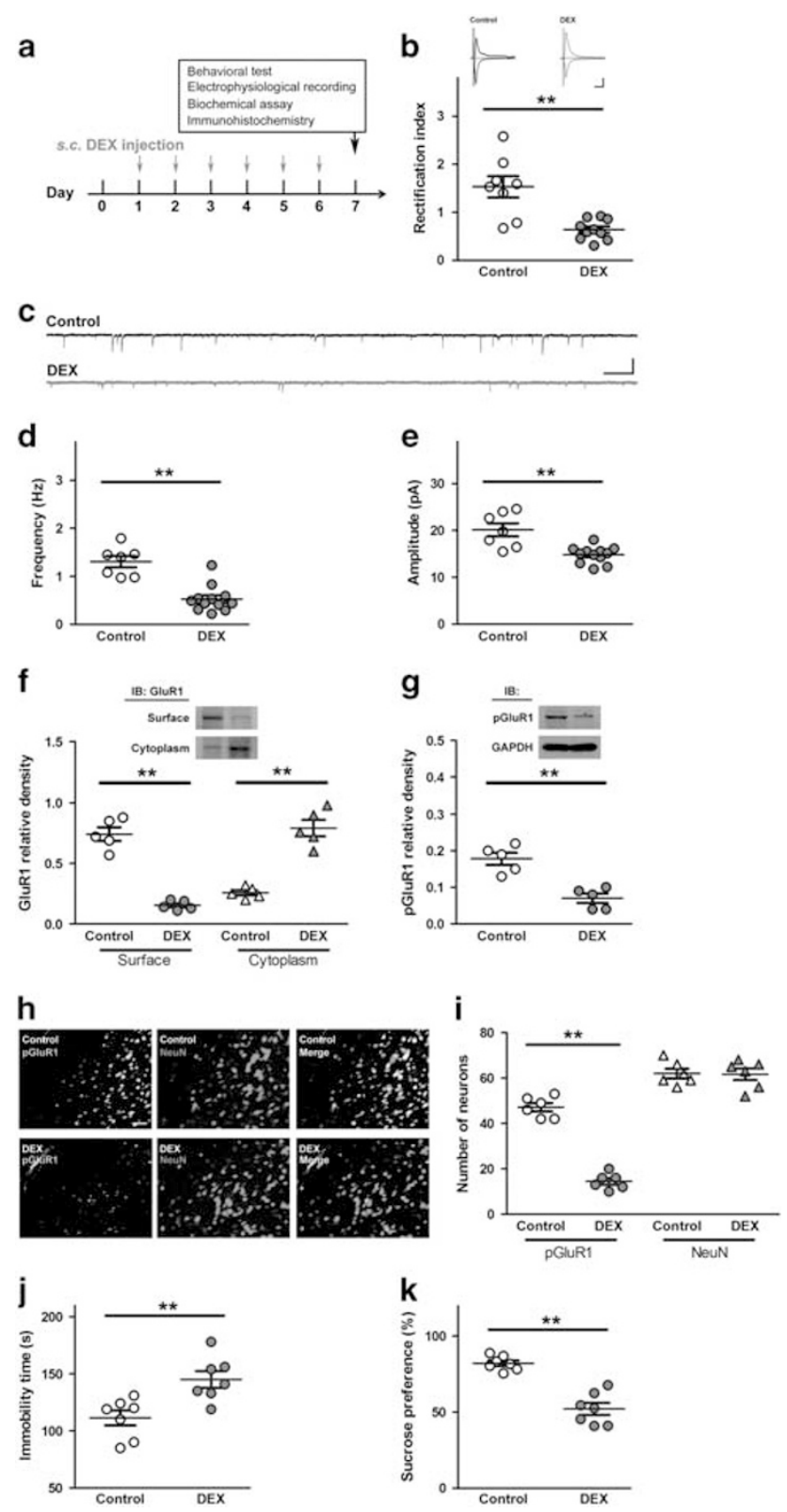

Figure 5 Glucocorticoid receptor activation mimics chronic stressinduced depression of excitatory synaptic transmission via both pre- and postsynaptic mechanisms. (a) Schematic experimental procedure of the effect of glucocorticoid receptor agonist, dexamethasone (DEX), in naive rats. (b) Rectification index of AMPA receptor obtained from control and DEX groups. Inset: represented traces of rectification index obtained from control $(n=8)$ and DEX $(n=10)$ groups. Calibration: 50 pA, 20 ms. (c) Representative traces of mEPSCs recorded from vIPAG neurons dissected from control and DEX groups. Calibration: 25 pA, 600 ms. (d, e) Vertical scatterplots depicting the distribution of frequency (d) and amplitude (e) of mEPSCs recorded from vIPAG neurons obtained from control $(n=7)$ and DEX $(n=12)$ groups. ( $f, g)$ Vertical scatterplot depicting the distribution of surface and cytoplasm (f) and pGluRI (g) expressions in the VIPAG. Inset: represented western blot of protein expression from control $(n=5)$ and DEX $(n=5)$ groups. ( $h, i)$ Immunohistochemistry images $(h)$ and quantitative analysis (i) of pGluRI and NeuN in the vIPAG slices dissected from control $(n=6)$ and DEX $(n=6)$ groups. Calibration: $50 \mu \mathrm{m}$. (j, k) Vertical scatterplot depicting the distribution of immobility time of tail suspension test (j) and sucrose consumption of sucrose preference test $(k)$ from control $(n=7)$ and DEX $(n=7)$ groups. Data are expressed as mean \pm SEM; $* * * 0.0$ I vs Control. 
from that in the control group. These results suggest that chronic stress does not affect GABAergic transmission in the vlPAG.

\section{DISCUSSION}

To the best of our knowledge, this is the first report to show that chronic stress suppresses glutamatergic transmission in the vlPAG and leads to depression-like maladaptive behavior through reduced glutamate release presynaptically and diminished GluR1 expression on the surface postsynaptically. Decreased excitatory transmission in the vlPAG contributes to the development of chronic stress-induced maladaptive behavior.

In contrast to defensive behaviors such as flight, the ventrolateral periaqueductal gray has been evidenced recently as a center controlling passive coping and depressionlike behaviors (Berton et al, 2007; Johnson et al, 2016; Landgraf et al, 2016; Wang et al, 2016). Previously, Berton et al (2007) found that local overexpression of deltaFosB in the vlPAG dramatically reduces inescapable shock-induced depression-like behavior. This hints that the vlPAG actually controls stress-induced despair behavior. A recent report notes that a loss of circadian rhythms in the PAG is associated with LH-induced depression-like behavior, demonstrating that the PAG is a brain region directly involved in the development of behaviors associated with helplessness (Landgraf et al, 2016). Another study demonstrates that knockdown of galanin receptor 1 specifically in the vlPAG rescues chronic mild stress-induced depression-like behavior (Wang et al, 2016). This implies that the vlPAG might represent a novel brain region for controlling chronic stressinduced depression-like behavior. Moreover, inactivation of the bed nuclei of the stria terminalis-vlPAG pathway increases immobility time in tail suspension and forced swim tests, suggesting that the vlPAG serves as a core that modulates passive coping behavior (Johnson et al, 2016). Taken together, all of the above evidence demonstrates that the vlPAG is a critical brain nucleus governing stressinduced depression-like behavior. In the current study, we found that the expression level of GluR1 in the vlPAG correlated negatively with $\mathrm{LH}$-induced depression-like behavior. Local pharmacological inhibition of GluR1 in the vlPAG leads to depression-like behavior. Interestingly, intradorsal PAG microinjections of glutamatergic agonists have been shown to enhance conditioned freezing and promote proaversive effects (Reimer et al, 2012). Another study demonstrates that activation of glutamatergic transmission in the dorsolateral PAG enhances the flight reaction (Moreira et al, 2004). This body of evidence suggests that glutamatergic transmission in the dorsal PAG is crucial for the regulation of freezing, flight, and aversive behaviors. However, although it is still unclear whether alteration of GluR1 regulates freezing, flight, and aversion, these behaviors are known to be controlled by the vlPAG too. Therefore, our results that the vlPAG governs chronic stress-induced depression-like behavior might provide a new insight into the pathophysiology of depression and an unexplored aspect of study about psychiatric-related behaviors.
Numerous studies suggest that chronic stress induces divergent synaptic plasticity in different brain regions (Hajszan et al, 2009; Kallarackal et al, 2013; Li et al, 2011; Penzo et al, 2014; Wei et al, 2016; Yuen et al, 2012). Repeated stress leads to the loss of glutamatergic transmission including reduction in presynaptic glutamate release and postsynaptic GluR1 in the PFC, hence resulting in object recognition memory impairment (Yuen et al, 2012). Moreover, chronic unpredictable stress elicits depression-like affective state because of diminishing GluR1-mediated synaptic transmission in the temporoammonic-CA1 synapse (Kallarackal et al, 2013). The PAG, a crucial midbrain region controlling susceptibility to stress and generation of fearrelated responses, is involved in stress-related psychiatric disorders (Norman et al, 2010; Wang et al, 2016). A previous study indicated that chronic restrain stress decreases excitatory amino acid transporter 2 protein level in the vlPAG; this implies that suppression of excitatory neurotransmission and neuronal dysfunction is involved in stressinduced neuropsychiatric disorders (Imbe et al, 2012). Therefore, in the current study, we first investigated the cellular mechanisms of neurotransmission in the vlPAG underlying chronic stress-induced depression-like maladaptive behavior. We found that chronic unpredictable foot shock stress elicits hypofunction of glutamatergic transmission through both pre- and postsynaptic mechanisms. Presynaptically, the projection fibers originating from other brain regions show decreased glutamate release onto vlPAG neurons. Postsynaptically, GluR1 on the cell surface endocytosed into cytoplasm, causing reduction in glutamatergic transmission in the vlPAG. The activity of glutamatergic transmission in the vlPAG not only affects nociceptive modulation (Ho et al, 2013, 2015), but also stress-associated defensive reaction and autonomic regulation (Tovote et al, 2016). Thus, the vlPAG plays an essential role in stressinduced maladaptive behavior and might be a new target brain region for the development of therapeutic strategy.

In addition to stress-induced depression-like maladaptive behavior, stress also affects pain sensation bidirectionally. Stress may either suppress or exacerbate pain sensation, termed stress-induced analgesia and stress-induced hyperalgesia, respectively, depending on the duration and intensity of the stressor. A previous report demonstrates that acute footshock stress increases paw withdrawal latency; this implies that acute stress may result in stress-induced analgesia (Donello et al, 2011). Another study reports that chronic footshock leads to visceral hyperalgesia, suggesting that chronic stressors may cause stress-induced hyperalgesia (Robbins and Ness, 2008). In the present study, we found that chronic stress reduced both paw withdrawal threshold and latency, that is, stress-induced hyperalgesia. These results are consistent with our previous reports showing that spinal nerve ligation induces mechanical allodynia (Ho et al, 2013, 2015). Actually, it is difficult to disentangle the relationship between pain and depression, as both pain and depression usually co-occur and share some similar symptoms, implying overlapping neurobiological underpinnings. Therefore, the comorbidity of pain and depression should be further investigated to reveal this issue. Furthermore, we could not exclude the possibility that acute footshock stress affects the cellular changes in the vlPAG and behavioral 
maladaptation. The neuroplastic and behavioral changes after acute footshock stress should also be further examined.

Activation of GR signaling in the brain in response to stress has been implicated in the pathogenesis of stressassociated psychiatric disorders (Wei et al, 2016; Yuen et al, 2012). Several lines of evidence demonstrate that stress induces neuroplastic changes in different brain regions through glucocorticoid-related cascades and downstream signaling affecting synaptic transmission and maladaptive behavior (Di et al, 2016; Licznerski et al, 2015; Yuen et al, 2012). A previous study indicated that repeated stress decreases glutamatergic transmission in the PFC contributing to the impairment of object recognition memory. The impairments of cognitive processes are blocked and mimicked by GR antagonist and corticosterone, respectively, indicating that stress downregulates glutamatergic transmission through GR-dependent mechanisms (Yuen et al, 2012). A recent report demonstrated that $\mathrm{LH}$ paradigm significantly reduces serum and glucocorticoid-regulated kinase 1, a GRassociated kinase, protein expression in the PFC, associated with posttraumatic stress disorder, implying GR-mediated signaling plays a critical role in learned helplessness-induced depression-like behavior (Licznerski et al, 2015). In the current study, systemic injection of GR antagonist abolished chronic stress-induced suppression of glutamatergic transmission. Exogenous administration of GR agonist elicited similarly chronic stress-induced neuroplastic changes in the vlPAG of naive rats. Although high-dose GR agonist decreased body weight and locomotor activity, we could not exclude the possibility that the systemic effects of highdose GR agonist suppress glutamatergic transmission in the vlPAG. These results suggest chronic stress-induced neuroplastic changes and maladaptive behavior occur through GRmediated mechanisms.

In addition to the AMPA receptor, accumulating evidence has shown that the NMDA receptor and metabotropic glutamate receptors (mGluRs) are highly implicated in learned helplessness-induced psychopathology (Burgdorf et al, 2013; Pignatelli et al, 2013). Burgdorf et al (2013) demonstrate that an NMDA receptor glycine-site functional partial agonist rescues LH-induced depression-like behavior, implying NMDA receptor involvement in LH-induced depression-like behavior. In the present study, we found a reduced $\mathrm{EPSC}_{\mathrm{AMPA} / \mathrm{NMDA}}$ ratio in the vlPAG of $\mathrm{LH}$-induced rats, implying that chronic stress might cause an enhanced NMDA receptor-mediated current in the vlPAG. However, the detailed mechanisms of LH-induced upregulation of NMDA receptors in the vlPAG should be examined. Furthermore, mGluRs have also been reported to participate in LH-induced depression-like behavior, as enhanced expression and function of mGluR5 in the hippocampus is associated with the depression-like phenotype of LH rats (Pignatelli et al, 2013). In the LH paradigm, we could not exclude the possibility that LH-induced depression-like behavior is due to the alteration of mGluR in the vlPAG.

Multiple cell types in the vlPAG could be associated with a distinct behavioral phenotype (Bobeck et al, 2014; Tovote et al, 2016). A previous report demonstrates that inhibition of the GABAergic neurons in the vlPAG leads to muscle atonia, implying that vlPAG suppresses rapid eye movement sleep by providing GABAergic inhibition to rapid eye movement-promoting neurons (Weber et al, 2015). A recent study reports that activation of glutamatergic neurons in the vlPAG specifically triggers freezing behavior, suggesting that some of the glutamatergic vlPAG neurons are positively correlated with freezing behavior (Tovote et al, 2016). In the current study, however, whether or not the cell type specificity in the vlPAG governs chronic stress-induced depression-like maladaptive behavior is still uncertain and should be further investigated.

The imbalances of excitatory and inhibitory neurotransmission in the brain regions have been evidenced as possible pathological mechanisms of neuropsychiatric disorders (Ghosal et al, 2017). Defects in GABAergic transmission have been reported to contribute to the etiology of major depressive disorder (Luscher and Fuchs, 2015). Mice with $\gamma 2$ subunit-lacking $\mathrm{GABA}_{\mathrm{A}}$ receptors in the forebrain exhibit core symptoms of behavioral despair and hypothalamicpituitary-adrenal axis hypersensitivity, demonstrating that GABAergic deficits contribute to depression-like behavior (Shen et al, 2010). A recent study also revealed that downregulation of glutamatergic transmission owing to stress-induced GABAergic deficits is involved in depression-like brain states, providing novel mechanisms of etiology of major depressive disorder (Ren et al, 2016). Mounting evidence has demonstrated that deficits in the GABAergic-mediated system play a pivotal role in major depressive disorder. However, we found no significant changes in GABAergic transmission in the vlPAG after chronic stress paradigm in the current study. This discrepancy may be because of the following possibilities. First, insufficient GABAergic-mediated responses might be recruited originating from other brain nuclei. Neurons in the vlPAG receive GABAergic inputs from both local interneurons (Aubrey et al, 2017; Lau and Vaughan, 2014) and the central amygdala projection (CeL) (Penzo et al, 2014). A previous study indicated that foot shock potentiates excitatory synapses onto somatostatin-positive neurons in the $\mathrm{CeL}$, sending direct long-range GABAergic projections onto vlPAG neurons (Penzo et al, 2014). However, in the present study, we did not find hyperactivity of GABAergic transmission in the vlPAG after the foot shock paradigm. This may be because the long-range GABAergic projections from the CeL might not be intact and sufficient for recruitment; hence, no deficits of the GABAergic system were observed in our results. Second, different stress paradigms may cause distinct effects on the glutamatergic and GABAergic systems. Previous reports demonstrate that diverse chronic pain models elicit neuroplastic changes selectively not only in the glutamatergic (Ho et al, 2015) but also GABAergic system (Tonsfeldt et al, 2016), implying various types of stress may affect different systems.

In summary, our study provides evidence that chronic foot shock exposure leads to depression-associated behavioral despair and anhedonia. Because of the complex nature of depression with altered neurotransmission across several brain regions, deficits in excitatory glutamatergic transmission in the vlPAG might be an underlying mechanism of chronic stress-elicited neuropsychiatric disorder-associated maladaptive behavior. Understanding the pathophysiological mechanisms involved in the expression of maladaptive behavior could be highly beneficial in fostering the development of novel therapeutic strategies for neuropsychiatric disorders. 


\section{FUNDING AND DISCLOSURE}

The authors declare no conflict of interest.

\section{ACKNOWLEDGMENTS}

This work was supported by the Ministry of Science and Technology, Taipei, Taiwan: MOST 105-2628-B-715-003MY3, 104-2320-B-715-004-MY3, NSC 102-2628-B-715-001, 101-2320-B-715-001-MY3, and MOST 105-2320-B-715-003MY2 to H-YP and Y-CH; by the Mackay Memorial Hospital MMH-MM-10206, MMH-MM-10302, MMH-MM-10403, and MMH-MM-10503 to H-YP; as well as by the Department of Medicine, Mackay Medical College 1001A03, 1001B07, 1011B02, 1021B08, 1031A01, 1031B07, 104B06, $1042 \mathrm{~A} 08,1051 \mathrm{~B} 03$, and 1051B04 to $\mathrm{H}-\mathrm{YP}$ and $\mathrm{Y}-\mathrm{CH}$.

\section{REFERENCES}

Aubrey KR, Drew GM, Jeong HJ, Lau BK, Vaughan CW (2017). Endocannabinoids control vesicle release mode at midbrain periaqueductal grey inhibitory synapses. J Physiol 595: 165-178.

Auer DP, Putz B, Kraft E, Lipinski B, Schill J, Holsboer F (2000). Reduced glutamate in the anterior cingulate cortex in depression: an in vivo proton magnetic resonance spectroscopy study. Biol Psychiatry 47: 305-313.

Berton O, Covington HE 3rd, Ebner K, Tsankova NM, Carle TL, Ulery $\mathrm{P}$ et al (2007). Induction of deltaFosB in the periaqueductal gray by stress promotes active coping responses. Neuron 55: 289-300.

Bobeck EN, Chen Q, Morgan MM, Ingram SL (2014). Contribution of adenylyl cyclase modulation of pre- and postsynaptic GABA neurotransmission to morphine antinociception and tolerance. Neuropsychopharmacology 39: 2142-2152.

Burgdorf J, Zhang XL, Nicholson KL, Balster RL, Leander JD, Stanton PK et al (2013). GLYX-13, a NMDA receptor glycine-site functional partial agonist, induces antidepressant-like effects without ketamine-like side effects. Neuropsychopharmacology 38: 729-742.

Di S, Itoga CA, Fisher MO, Solomonow J, Roltsch EA, Gilpin NW et al (2016). Acute stress suppresses synaptic inhibition and increases anxiety via endocannabinoid release in the basolateral amygdala. J Neurosci 36: 8461-8470.

Donello JE, Guan Y, Tian M, Cheevers CV, Alcantara M, Cabrera S et al (2011). A peripheral adrenoceptor-mediated sympathetic mechanism can transform stress-induced analgesia into hyperalgesia. Anesthesiology 114: 1403-1416.

Duman RS, Aghajanian GK, Sanacora G, Krystal JH (2016). Synaptic plasticity and depression: new insights from stress and rapid-acting antidepressants. Nat Med 22: 238-249.

Fava M (2003). Diagnosis and definition of treatment-resistant depression. Biol Psychiatry 53: 649-659.

Gerhard DM, Wohleb ES, Duman RS (2016). Emerging treatment mechanisms for depression: focus on glutamate and synaptic plasticity. Drug Discov Today 21: 454-464.

Ghosal S, Hare B, Duman RS (2017). Prefrontal cortex GABAergic deficits and circuit dysfunction in the pathophysiology and treatment of chronic stress and depression. Curr Opin Behav Sci 14: $1-8$.

Hajszan T, Dow A, Warner-Schmidt JL, Szigeti-Buck K, Sallam NL, Parducz A et al (2009). Remodeling of hippocampal spine synapses in the rat learned helplessness model of depression. Biol Psychiatry 65: 392-400.

Heinricher MM, Tavares I, Leith JL, Lumb BM (2009). Descending control of nociception: Specificity, recruitment and plasticity. Brain Res Rev 60: 214-225.
Ho YC, Cheng JK, Chiou LC (2013). Hypofunction of glutamatergic neurotransmission in the periaqueductal gray contributes to nerve-injury-induced neuropathic pain. J Neurosci 33: 7825-7836.

Ho YC, Cheng JK, Chiou LC (2015). Impairment of adenylyl cyclase-mediated glutamatergic synaptic plasticity in the periaqueductal grey in a rat model of neuropathic pain. $J$ Physiol 593: 2955-2973.

Ho YC, Lee HJ, Tung LW, Liao YY, Fu SY, Teng SF et al (2011). Activation of orexin 1 receptors in the periaqueductal gray of male rats leads to antinociception via retrograde endocannabinoid (2-arachidonoylglycerol)-induced disinhibition. J Neurosci 31: $14600-14610$.

Imbe H, Kimura A, Donishi T, Kaneoke Y (2012). Chronic restraint stress decreases glial fibrillary acidic protein and glutamate transporter in the periaqueductal gray matter. Neuroscience 223: 209-218.

Johnson SB, Emmons EB, Anderson RM, Glanz RM, Romig-Martin SA, Narayanan NS et al (2016). A basal forebrain site coordinates the modulation of endocrine and behavioral stress responses via divergent neural pathways. J Neurosci 36: 8687-8699.

Kallarackal AJ, Kvarta MD, Cammarata E, Jaberi L, Cai X, Bailey AM et al (2013). Chronic stress induces a selective decrease in AMPA receptor-mediated synaptic excitation at hippocampal temporoammonic-CA1 synapses. J Neurosci 33: 15669-15674.

Krystal JH, Sanacora G, Duman RS (2013). Rapid-acting glutamatergic antidepressants: the path to ketamine and beyond. Biol Psychiatry 73: 1133-1141.

Landgraf D, Long JE, Welsh DK (2016). Depression-like behaviour in mice is associated with disrupted circadian rhythms in nucleus accumbens and periaqueductal grey. Eur $J$ Neurosci 43: 1309-1320.

Lau BK, Vaughan CW (2014). Descending modulation of pain: the GABA disinhibition hypothesis of analgesia. Curr Opin Neurobiol 29: 159-164.

Lee HJ, Chang LY, Ho YC, Teng SF, Hwang LL, Mackie K et al (2016). Stress induces analgesia via orexin 1 receptor-initiated endocannabinoid/CB1 signaling in the mouse periaqueductal gray. Neuropharmacology 105: 577-586.

Lee HK, Takamiya K, Han JS, Man H, Kim CH, Rumbaugh G et al (2003). Phosphorylation of the AMPA receptor GluR1 subunit is required for synaptic plasticity and retention of spatial memory. Cell 112: 631-643.

Li B, Piriz J, Mirrione M, Chung C, Proulx CD, Schulz D et al (2011). Synaptic potentiation onto habenula neurons in the learned helplessness model of depression. Nature 470: 535-539.

Licznerski P, Duric V, Banasr M, Alavian KN, Ota KT, Kang HJ et al (2015). Decreased SGK1 expression and function contributes to behavioral deficits induced by traumatic stress. PLoS Biol 13: e1002282.

Luscher B, Fuchs T (2015). GABAergic control of depressionrelated brain states. Adv Pharmacol 73: 97-144.

Luscher B, Shen Q, Sahir N (2011). The GABAergic deficit hypothesis of major depressive disorder. Mol Psychiatry 16: 383-406.

Maier SF (1984). Learned helplessness and animal models of depression. Prog Neuropsychopharmacol Biol Psychiatry 8: 435-446.

Martinez-Rivera A, Hao J, Tropea TF, Giordano TP, Kosovsky M, Rice RC et al (2017). Enhancing VTA Cav1.3 L-type Ca2+ channel activity promotes cocaine and mood-related behaviors via overlapping AMPA receptor mechanisms in the nucleus accumbens. Mol Psychiatry (doi:10.1038/mp.2017.9).

Moreira FA, Molchanov ML, Guimaraes FS (2004). Ionotropic glutamate-receptor antagonists inhibit the aversive effects of nitric oxide donor injected into the dorsolateral periaqueductal gray of rats. Psychopharmacology 171: 199-203.

Nishitani N, Nagayasu K, Asaoka N, Yamashiro M, Shirakawa H, Nakagawa $\mathrm{T}$ et al (2014). Raphe AMPA receptors and nicotinic 
acetylcholine receptors mediate ketamine-induced serotonin release in the rat prefrontal cortex. Int J Neuropsychopharmacol 17: 1321-1326.

Norman GJ, Karelina K, Zhang N, Walton JC, Morris JS, Devries AC (2010). Stress and IL-1beta contribute to the development of depressive-like behavior following peripheral nerve injury. $\mathrm{Mol}$ Psychiatry 15: 404-414.

Ossipov MH, Dussor GO, Porreca F (2010). Central modulation of pain. J Clin Invest 120: 3779-3787.

Park P, Sanderson TM, Amici M, Choi SL, Bortolotto ZA, Zhuo M et al (2016). Calcium-permeable AMPA receptors mediate the induction of the protein kinase A-dependent component of longterm potentiation in the hippocampus. J Neurosci 36: 622-631.

Penzo MA, Robert V, Li B (2014). Fear conditioning potentiates synaptic transmission onto long-range projection neurons in the lateral subdivision of central amygdala. J Neurosci 34: 2432-2437.

Pignatelli M, Vollmayr B, Richter SH, Middei S, Matrisciano F, Molinaro G et al (2013). Enhanced mGlu5-receptor dependent long-term depression at the Schaffer collateral-CA1 synapse of congenitally learned helpless rats. Neuropharmacology 66: 339-347.

Reimer AE, de Oliveira AR, Brandao ML (2012). Glutamatergic mechanisms of the dorsal periaqueductal gray matter modulate the expression of conditioned freezing and fear-potentiated startle. Neuroscience 219: 72-81.

Ren Z, Pribiag H, Jefferson SJ, Shorey M, Fuchs T, Stellwagen D et al (2016). Bidirectional homeostatic regulation of a depressionrelated brain state by gamma-aminobutyric acidergic deficits and ketamine treatment. Biol Psychiatry 80: 457-468.

Robbins MT, Ness TJ (2008). Footshock-induced urinary bladder hypersensitivity: role of spinal corticotropin-releasing factor receptors. J Pain 9: 991-998.

Shen Q, Lal R, Luellen BA, Earnheart JC, Andrews AM, Luscher B (2010). gamma-Aminobutyric acid-type A receptor deficits cause hypothalamic-pituitary-adrenal axis hyperactivity and antidepressant drug sensitivity reminiscent of melancholic forms of depression. Biol Psychiatry 68: 512-520.

Shi SH, Hayashi Y, Petralia RS, Zaman SH, Wenthold RJ, Svoboda $\mathrm{K}$ et al (1999). Rapid spine delivery and redistribution of AMPA receptors after synaptic NMDA receptor activation. Science 284: 1811-1816.

Tonsfeldt KJ, Suchland KL, Beeson KA, Lowe JD, Li MH, Ingram SL (2016). Sex differences in GABAA signaling in the periaqueductal gray induced by persistent inflammation. I Neurosci 36: 1669-1681.

Tovote P, Esposito MS, Botta P, Chaudun F, Fadok JP, Markovic M et al (2016). Midbrain circuits for defensive behaviour. Nature 534: 206-212.

Vianna DM, Graeff FG, Brandao ML, Landeira-Fernandez J (2001). Defensive freezing evoked by electrical stimulation of the periaqueductal gray: comparison between dorsolateral and ventrolateral regions. Neuroreport 12: 4109-4112.

Wang P, Li H, Barde S, Zhang MD, Sun J, Wang T et al (2016). Depression-like behavior in rat: involvement of galanin receptor subtype 1 in the ventral periaqueductal gray. Proc Natl Acad Sci USA 113: E4726-E4735.

Weber F, Chung S, Beier KT, Xu M, Luo L, Dan Y (2015). Control of REM sleep by ventral medulla GABAergic neurons. Nature 526: 435-438.

Wei J, Xiong Z, Lee JB, Cheng J, Duffney LJ, Matas E et al (2016). Histone modification of Nedd4 ubiquitin ligase controls the loss of AMPA receptors and cognitive impairment induced by repeated stress. J Neurosci 36: 2119-2130.

Wohleb ES, Franklin T, Iwata M, Duman RS (2016). Integrating neuroimmune systems in the neurobiology of depression. Nat Rev Neurosci 17: 497-511.

Yuen EY, Wei J, Liu W, Zhong P, Li X, Yan Z (2012). Repeated stress causes cognitive impairment by suppressing glutamate receptor expression and function in prefrontal cortex. Neuron 73: 962-977.

Supplementary Information accompanies the paper on the Neuropsychopharmacology website (http://www.nature.com/npp) 